\title{
COUPLING A LINEAR PRESSURE AND MASS FLOW FREQUENCY MODEL OBTAINED FROM MEASUREMENTS AT THE INTAKE OF AN IC ENGINE WITH A NON-LINEAR TIME DOMAIN CODE
}

\author{
David Chalet \\ LUNAM Université, Ecole Centrale de Nantes, \\ LHEEA UMR CNRS 6598 \\ Nantes, France
}

Haitham Mezher ${ }^{1}$

LUNAM Université, Ecole Centrale de Nantes, LHEEA UMR CNRS 6598

Nantes, France

${ }^{1}$ Contact Author

\author{
Jérôme Migaud \\ Advanced Development, Mann+Hummel \\ Laval, France
}

\section{ABSTRACT}

Engine simulation software has become synonymous with automotive design and component development. An integral part of any engine simulation is the correct modeling of the air flow at the intake. This air flow, which is highly compressible and unsteady, has a first order influence on the trapped air mass inside the cylinder and therefore on the behavior of the engine (torque response and emissions). The non-linear modeling of the air paths at the intake is done using a space-time meshing and by solving the $1 \mathrm{D}$ equations with a proper time scheme. Such methods are the bases of today's engine simulation codes [1]. The main constraint with these methods is the time needed to model complex geometries, whether being the simulation time or the time spent on calibrating the said models with experimental measurements. These complicated geometries become problematic to accurately predict, particularly the charge air cooler $(\mathrm{CAC})$ which is responsible for cooling the air flow on a turbocharged engine

Another approach is to use frequency domain models to describe the fluctuating pressure and mass flow [2]. Although this approach is simpler, faster in terms of computing time and offers many experimental techniques to characterize complicated geometries; important limitations can appear when it is confronted to the effects of high pressure levels and pulsating mass flow. Furthermore, the models behind such methods are designed to be used in the frequency domain, contrary to an engine simulation that works solely with time domain variables.

In this article, a linear frequency domain model known as a transfer matrix is used. This concept is nothing new in acoustics; however here it was developed by experimentally measuring the transfer matrix [3] for a simple tube on a dedicated bench under conditions similar to those encountered on an engine [4]. The approach is then extended to measure the transfer matrix of a charge air cooler (CAC) on a real engine test bench. The measured discrete transfer matrix, defined in terms of fluctuating pressure and mass flow, is then transformed to a continuous frequency model and coded in Simulink ${ }^{\circledR}$. The latter is coupled to the non-linear engine simulation software GT-Power $\AA$.

The objective is to accurately model the pressure and mass flow of a complicated geometry using experimental measurements and a linear frequency model then to couple the transfer matrix to an engine simulation code, thus replacing the need for a meshed model.

\section{INTRODUCTION}

With ever more growing needs for fuel consumption savings and future $\mathrm{CO}_{2}$ legislations, $1 \mathrm{D}$ engine simulation models have become an intrinsic part of any OEM or engine manufacturer. Engine simulation software can be split up into two main categories: high resolution codes that track instantaneous engine parameters such as intake pressure and mass flow; and mean value resolution codes that calculate mean engine behavior such as fuel consumption over a driving cycle, for instance, or torque and horsepower data. High resolution codes are based on a non-linear description of the gas dynamics equations and provide an important level of accuracy by enabling a close inspection of the fluctuating variables. However, they can be far too time consuming if used for an entire engine driving cycle. 
Furthermore, 1D tools that offer such a high level of fidelity require a detailed modeling of the engine particularly the air intake line [1] [5] and the results are dependent on the tools used. Complicated air intake parts on a modern turbocharged engine are problematic to model because of their geometries. The latter can depict multi-dimensional effects due to bends, changes in section and high mass flow rates. FIGURE 1 shows the air path of a turbocharged engine with a picture of a charge air cooler.

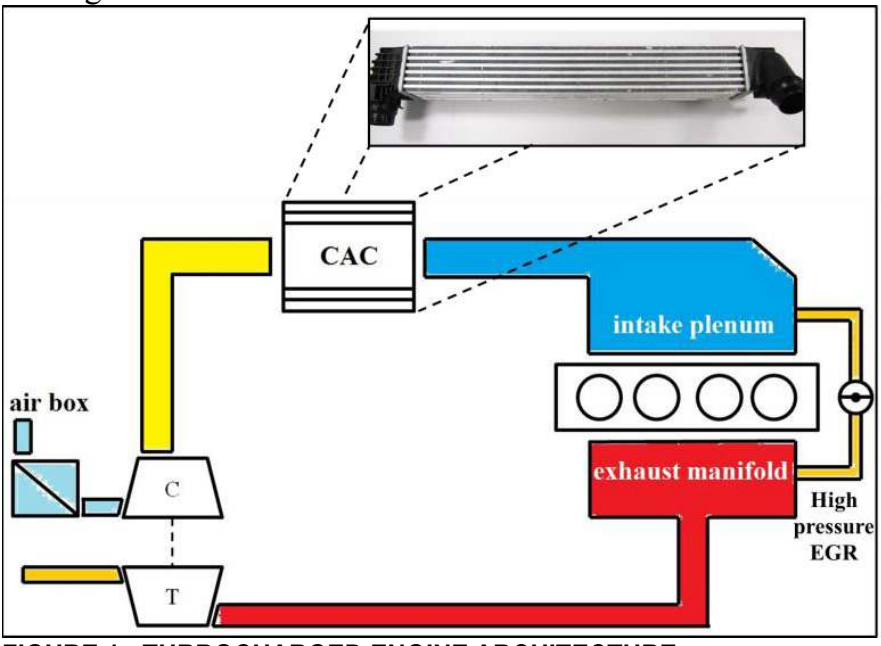

FIGURE 1 - TURBOCHARGED ENGINE ARCHITECTURE

The charge air cooler (CAC), is a rather complicated geometry that is made up of a series of multi-tubes between two volumes. The CAC's main role is to cool the pressurized air exiting the compressor in order to increase its density and therefore there is more air available to the engine for combustion. This element introduces a pressure drop to the intake line but also interacts with the dynamic pressure and mass flow and disturbs the wave action upstream and downstream of the pipes. An accurate modeling of the CAC is essential in order to correctly predict intake pressure and consequent engine characteristics. Benson [6] modeled the $\mathrm{CAC}$ as a simple tube between two boundary conditions, his method takes into account the length of the CAC but disregards the damping effects due to the inner volume of the element. Seifert [7] proposed to model the CAC as a series of parallel tubes linked together with flow-splitting junctions at the inlet and outlet of the element. This type of approach is one of the most employed today [8] to model wave action through heat exchangers and CACs. Although this type of non-linear approach yields reliable results, the final model have to be calibrated with experimental measurements for pressure loss coefficients and corrected lengths and sections in order to achieve the correct dynamic effects as those encountered experimentally. This means that there is a final calibration phase where the model is "tweaked" against experimental engine test bench results to insure fidelity. Non-linear quasi-3D and fully 3D solutions can be used to calculate the dynamic behavior of complicated parts without the need to special calibration process of the models. Montenegro et al. [9] [10] compared non-linear 1D methods to quasi-3D approaches where higher order modes where correctly represented with acceptable simulation time. Although good simulation results are obtained using similar techniques, it remains difficult to model an entire engine wave action and gas exchanges using such tools due to the computational time needed.

Linear methods for computing gas dynamics also exist. Wallace [11] compared results obtain with the linear approximation to those obtained with the method of characteristics and Chen et al. [12] compared various linear models to non-linear ones. It was found that the linear modeling could be applicable to the intake line with good assumptions, however errors would arise when using linear approaches at the exhaust due to the high temperatures [13]. Pearson et al. [14] used a wave tracking technique to quickly predict the geometry of the intake line at the early stages of the design. Linear approaches are faster and easier to implement, their main drawback is the lack of precision when compared to non-linear modeling. For this reason they are often set aside and the entire engine (both intake and exhaust) is modeled using non-linear discretization of the space-time domains to the detriment of computing time.

That being said, linear calculations are still used with success to study pressure and mass flow fluctuations associated with higher frequencies, mainly for acoustic studies and noise cancellation [15] [16]. This is because usually the radiated noise from the engine's intake or exhaust is generally due to relatively small pressure and mass flow perturbations so the acoustic theory can be applied. This is done in the frequency domain where the time domain signals are treated in order to access their frequency spectrum. This frequency approach makes it easier to achieve acoustic studies, with the introduction of concepts such as impedance or transfer matrices [2]. Linear acoustic theory becomes challenging to use when it is applied to directly model elements that make up an air intake line, such as a turbocharger or a charge air cooler. This is due to the presence of a non-negligible Mach number inside the pipes that changes the Helmholtz equation [17] and introduces coupled effects between the pressure and the mass flow. A number of authors have studied acoustic modeling for air intake parts, using various simulation and measurement techniques. Knutsson et al. [18] used 3D CFD calculations for a charge air cooler to derive acoustic transfer matrices for this complicated geometry. Tikoja [19] presented a measurement technique for identifying the transfer matrix of a turbocharger. Portier et al. [20] introduced the measured transfer matrix of a turbocharger into a linear acoustic simulation code, thus removing the need to model this element.

Linear theory isn't solely used for acoustic prediction; it can be applied to determine the natural frequencies of the column of air present at the intake (Ohata et al. [21], Matsumoto et al. [22] and Winterbone et al. [23]) in order to tune the intake line to achieve resonant effects and enhance the volumetric efficiency. It can also be used for engine simulation purposes, although much more delicate in this form. Harrison et $a l$. [24] measured the impedance at the intake of the engine and 
proposed an engine simulation for a single cylinder engine using linear acoustic theory. The goal of the simulation was engine filling and volumetric efficiency prediction. It was found that an inertial corrective term had to be added to the equations in order to reach the proper pressure amplitudes. The difficulty of this method is to correctly describe the pressure response and the mass flow excitation: at the intake of the engine, the valves act as the acoustic source and create pressure and mass flow fluctuations. However this takes place with a mean flow that corresponds to the engine's aspiration. The dynamic fluctuations cannot be easily decoupled from the static components contrary to linear acoustics where the two are considered to be independent from one another.

It seems then, that a complete acoustic model wouldn't be able to achieve the same precision that is obtained using a full non-linear model due to these restrictions. An interesting point would be to model the valves (acoustic source and flow aspiration) in the non-linear domain thus making sure that the coupled effects are well described and keep a linear model for wave action for the rest of the intake or for a specific part.

Harrison et al. [25] and Sathyanarayana et al. [26] coupled an impedance model with the method of characteristics thus creating a hybrid boundary through which frequency domain data and time domain information can be exchanged. The results were improved compared to a fully acoustic model; however results were only presented in the frequency domain. Knutsson et al. [27] coupled a transfer matrix model to a nonlinear excitation in GT-Power. The transfer matrix was experimentally measured under zero mean flow conditions. The results were presented in the time and frequency domains; however the results lacked the precision needed for frequencies below $100 \mathrm{~Hz}$.

Fontana et al. [28] and Chalet et al. [4] [29] [30] presented a similar approach in its concept, where the impedance of the intake line is measured on a dedicated bench called "impulse flow bench". The specificity of this bench is that it enables a mass flow excitation of any intake line (or part) similar to the valve excitation at the intake of an engine. The technique gave good results and consisted of measuring the pressure response of the entire air intake line on the impulse flow bench. A transfer function (or impedance model) is then defined. This transfer function is coded in a coupled simulation with GTPower $\AA$. The latter provided the non-linear excitation, which is the excitation of the valves (or mass flow excitation across the valve) and the transfer function gave the pressure response upstream of the intake valve. FIGURE 2 presents the overall schematic of the technique where the intake line (in violet) is characterized in advance on the impulse flow bench in order to define a transfer function $T F$. This model replaces the 1D modeling of the intake line by providing the corresponding pressure response $p$ upstream of the intake valve (green point) using as input the non-linear mass flow rate $q m$ calculated by GT-Power ${ }^{\circledR}$. The latter is also used to model the moving parts (pistons, valves...) as well as the exhaust side.
The concept of using such technique is appealing to engine simulation because the simulation time is reduced on one hand and experimental characterization of the intake is directly used in the simulation on the other, which means that the time for setting up and calibrating the model is reduced. The difficulty is to take into account the coupled effects between the pulsating mass flow and the consequent pressure response specifically in the low frequency region. In fact, engine filling is a rather low frequency event that takes place at the corresponding harmonics of the engine speed, generally below $500 \mathrm{~Hz}$.

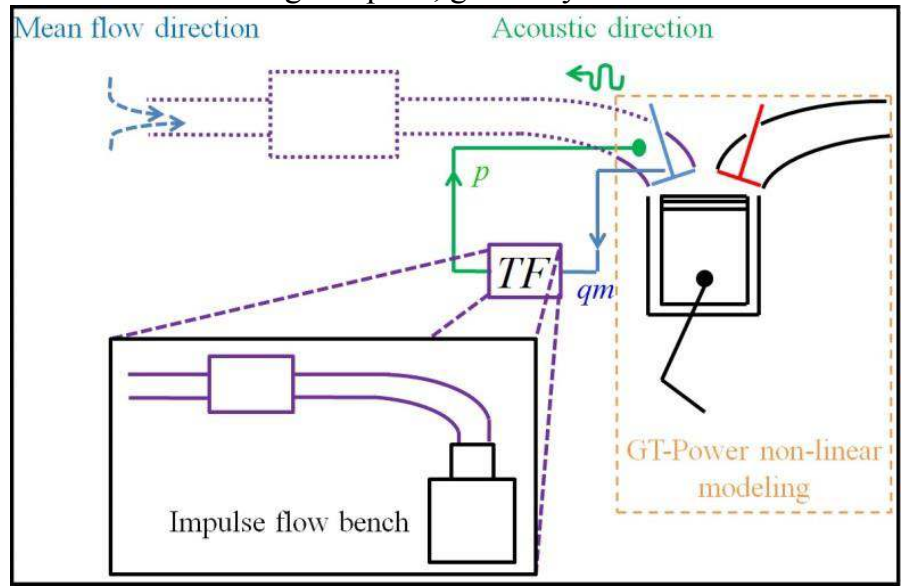

FIGURE 2 - REPRESENTATION OF THE TRANSFER FUNCTION [4]

The main inconvenience of the proposed transfer function method is that the entire air intake line has to be installed on the impulse flow bench in order to characterize it. Although this can be easily done for an intake line of a naturally aspirated engine, it would be more complicated for a turbocharged engine. This is because, such an intake line (FIGURE 1), present many thermodynamic discontinuities (turbocharger and charge air cooler) where different temperatures and boundary conditions can exist.

The goal of this paper is to split up the intake line into separate elements, using the same linear frequency domain approach on the impulse bench. This means that instead of using an impedance model defined upstream of the intake valve, a transfer matrix model is proposed. This has been already presented by Mezher et al. [3] [31] for a simple tube. It will be extended in this article to a more complicated geometry: the charge air cooler (CAC). The identified model will then be implemented in a coupled simulation with GT-Power ${ }^{\circledR}$.

Final validation will be a comparison between measured data and simulated ones in terms of pressure and mass flow, both in the frequency and time domains.

\section{TRANSFER FUNCTION AND MATRIX FOR A SIMPLE TUBE}

This paragraph will serve to explain and highlight the key aspects of identifying the transfer function and matrix for a simple tube [3] [31]. Absolute pressure $p_{\text {absolute }}$ can be decomposed into 2 parts: 


$$
p_{\text {absolute }}=p_{\text {mean }}+p
$$

Where $p_{\text {mean }}$ in equation (1) is the mean pressure and $p$ is the fluctuating pressure around the mean value or dynamic pressure. The idea is to linearly model the dynamic pressure while keeping a standard pressure loss model for the mean value. First a simple tube is mounted on the impulse dynamic bench, where an extremely rapid $(0.5 \mathrm{~ms})$ mass flow excitation $q m_{e x c}$ gives rise to pressure and mass flow response inside the tube. The pressure is recorded using Kistler 4005AA2R pressure sensors with a sampling frequency $F S=20 \mathrm{kHz}$. An impedance model or transfer function, is then defined. It is given by equation (2).

$$
T F(j \omega)=\frac{F F T(p)}{F F T\left(q m_{e x c}\right)}=\frac{P(j \omega)}{Q m_{e x c}(j \omega)}
$$

The rapid excitation profile makes it possible to directly identify the dynamic behavior of the column of air inside the tube using its resonance modes " $i$ " or resonant frequencies. This is done by determining a continuous model according to equation (3) where a set of 3 parameters $\left(X_{i}, \omega_{i}\right.$ and $\left.c_{i}\right)$ is identified for each resonant mode $i$.

$$
T F(s)=\frac{P(s)}{Q m_{\text {exc }}(s)}=\sum_{i=1}^{n} \frac{X_{i} s}{\left(\frac{s}{\omega_{i}}\right)^{2}+\frac{2{ }_{i} s}{i_{i}}+1}
$$

Equation (3) is an impedance model that links the pressure response to the impulse excitation mass flow. $X_{i}$ is a ram effect parameter that determines the energy available after excitation. $\omega_{i}$ is the angular frequency that depends on the resonant frequencies of the system and $\epsilon_{i}$ is a damping coefficient that models losses and viscous dissipation through propagation. This is bases of the method proposed by Chalet $e t$ al. [4] [29] [30]. Each tested geometry would have a different set of parameters and therefore would be characterized on the impulse bench. This means that for an entire air intake line, any changes made to a single component of the line would require a new identification on the bench. For this reason, the interest is turned to a transfer matrix model as it is defined by equation (1) and schematically explained in FIGURE 3.

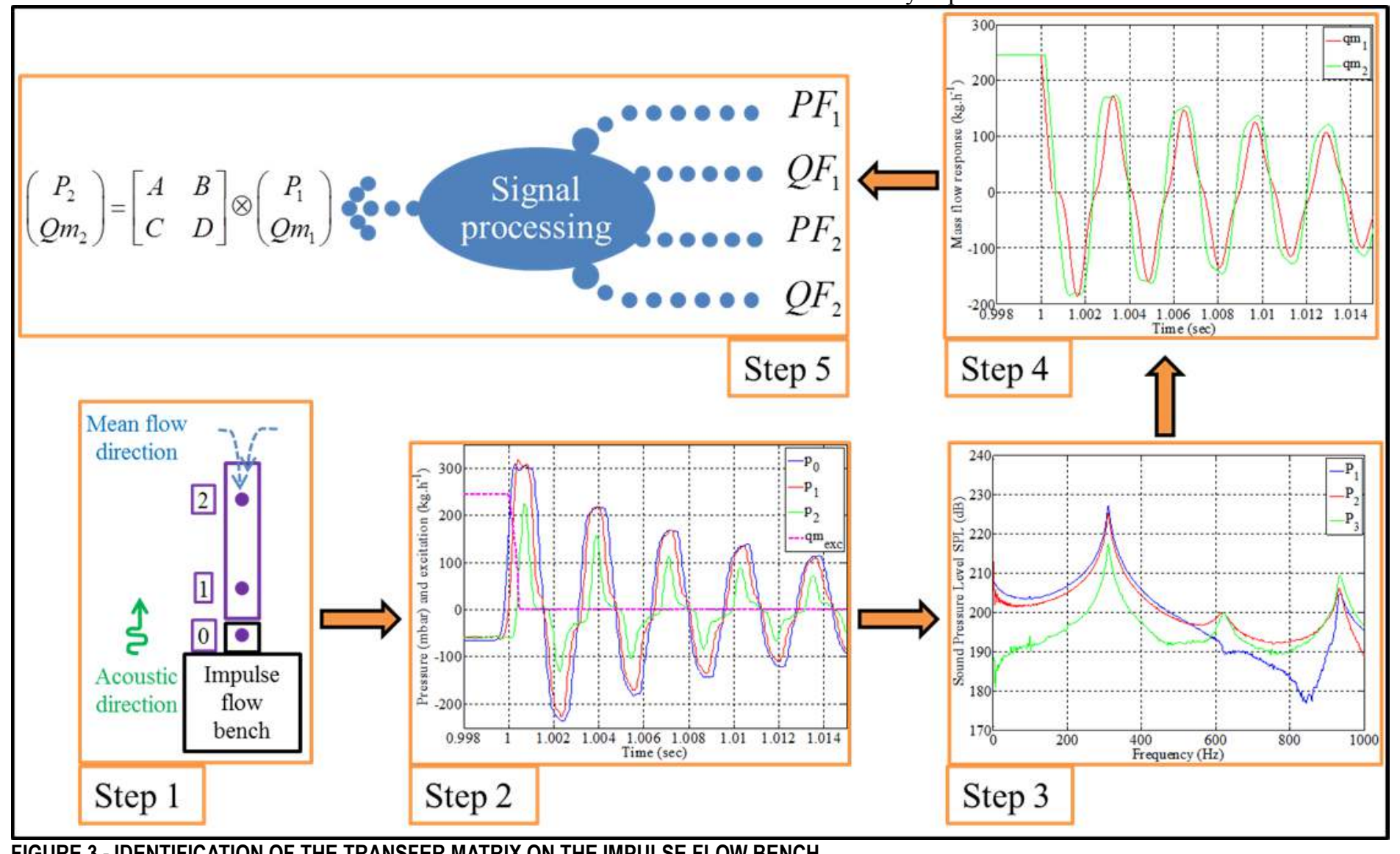

FIGURE 3 - IDENTIFICATION OF THE TRANSFER MATRIX ON THE IMPULSE FLOW BENCH 
The choice of a transfer matrix makes it possible to decompose the intake line into separate elements. The difficulty is defining a linear model that would be accurate enough to be used for engine simulation, i.e. for the low frequency region where the aero-acoustic effects of pressure and mass flow are highly coupled.

$$
\left(\begin{array}{c}
P_{2} \\
Q m
\end{array}\right)=\left[\begin{array}{ll}
A & B \\
C & D
\end{array}\right]_{j \omega} \otimes\left(\begin{array}{c}
P_{1} \\
\\
1
\end{array}\right)_{j \omega}
$$

The first step is defining the transfer matrix for a simple tube: a $185 \mathrm{~mm}$ tube equipped with 2 pressure sensors is chosen. Once this transfer matrix is validated, it will be used to experimentally measure the transfer matrix of a more complicated geometry, the charge air cooler.

The transfer matrix calculation for the tube is a 5 step process as highlighted by FIGURE 3:

STEP1: The tube equipped with two pressure sensors is mounted on the impulse flow bench via an adaptive part. A steady mass flow of $245 \mathrm{~kg} \cdot \mathrm{h}^{-1}$ is aspirated though the tube then extinguished in $0.5 \mathrm{~ms}$.

STEP2: The result is a hammer-like effect and a pressure response recorded with the three pressure sensors. $q m_{e x c}$ is the excitation profile plotted alongside the pressure traces.

STEP3: The pressure traces are filtered and transposed into the frequency domain. This gives access to the resonance modes at each point $p_{x, i}$ where $x$ is the abscissa of the tube and $i$ the harmonic number. The absolute pressure at each point is thus given by equation (2) where $n$ is the number of identified harmonics in the frequency range of interest.

$$
\left.p_{\text {absolute }}=p_{\text {mean }_{x}} \quad \sum_{i=1}^{n} p_{x, i}\right)
$$

In this case, $n=2(2$ peaks) between 0 and $1000 \mathrm{~Hz}$. A transfer function $P F_{x}$ is identified at each point; it is similar in form to equation (3). The transfer functions $P F_{1}$ and $P F_{2}$ are particularly interesting because they characterize the measurement points of the $185 \mathrm{~mm}$ tube.

STEP4: Once the dynamic behavior of the pressure response is identified, the mass flow data is calculated because it would be difficult to experimentally measure the instantaneous mass flow on the bench. This is done by simultaneously solving in the frequency domain the linearized continuity and momentum equations given by equations (3) and (4).

$$
P_{x} s \quad Z c_{0} \frac{d Q m_{x}}{d x}=0
$$

$$
\frac{1}{S} Q m_{x} s+\frac{d P_{x}}{d x}=0
$$

$Z_{c}$ is the pipe's characteristic impedance given by equation (5) and $S$ the cross section.

$$
Z_{c}=\frac{c_{0}}{S}
$$

Knowing $P_{x}$ from measurements, equations (6) and (7) are solved for $Q m_{x}$. Two additional transfer functions are then defined: $Q F_{1}$ and $Q F_{2}$, they characterize the mass flow response following the excitation of the bench.

STEP5: 4 transfer functions for pressure and mass flow have been identified for $x_{1}$ and $x_{2}$ of the tube. They represent a linear frequency domain characterization of the $185 \mathrm{~mm}$ tube. The transfer matrix given by equation (1) can now be identified. The tube is a symmetric and reciprocal geometry [32], this means that the determinant of the system (1) is equal to unity and that $A=D$. The transfer functions are fed into a Simulink routine where a dedicated signal processing program is applied. It consists of exciting the transfer functions with a repetitive valve-like mass flow signal, thus creating the desired responses of pressure and mass flow. Finally the poles $A, B, C, D$ for the matrix of the tube are identified.

The $185 \mathrm{~mm}$ tube has two resonant frequencies (2 peaks in FIGURE 3). A set of additional tubes can be added to the configuration explained in STEP1 in order to enrich the frequency content of the transfer matrix. For each additional tube, the 5 steps are repeated; this adds resonant frequencies and leads to a more robust transfer matrix. The transfer matrix for the tube is validated in terms of propagation properties and mass flow coherence by Mezher et al. [3] [31] and compared to the "classic" acoustic solution. It was found that the identification of the impulse flow bench enables a transfer matrix that takes into account the aero-acoustic coupling between pressure and mass flow while keeping a linear solution. It is therefore valid for usage on a more complicated geometry such as a charge air cooler.

\section{TRANSFER MATRIX FOR THE CHARGE AIR COOLER}

\section{MEASUREMENTS}

As shown in FIGURE 1, the charge air cooler is located between the compressor and the intake valves. Before proposing a methodology for characterizing this element, it would be interesting to briefly study the gas dynamics involved. The CAC receives an acoustic excitation from the intake valves on one hand and from the compressor wheel on the other. The mean flow direction is from the compressor to the intake valves (filling of the engine). Rammal et al. [33] have decomposed the turbocharger behavior into two parts: 
below $1200 \mathrm{~Hz}$ where the turbocharger mainly behaves as a passive termination and above $1200 \mathrm{~Hz}$ where the turbocharger starts participating in the acoustic generation of propagative waves. This means that for the low frequency region (below $500 \mathrm{~Hz}$ ) concerned with engine simulation, the compressor can be thought of as being a passive element. The acoustic direction is thus from the intake valves towards the CAC.

Once these boundary conditions are decided, the transfer matrix of the CAC can now be measured. The method proposed by To et al. [34] for measuring the transfer matrix of an unknown system is employed as well as the two-source method highlighted by Munjal et al. [35]. The CAC, which is the unknown system in question, is placed between two tubes whose matrices are determined in advance, as explained in the previous section. FIGURE 4 shows the experimental setup where the measuring tubes upstream and downstream of the CAC are equipped with pressure sensors. Two configurations relative to the two-source method are used: direct (d) and reverse (r) where the source is inverted (or the CAC is inverted) in a way to solve the linear system given by equation (6).

$$
\begin{aligned}
& \left(\begin{array}{c}
P_{3 d} \\
Q m_{3 d}
\end{array}\right)=\left\lfloor\begin{array}{cc}
A_{s} & B_{s} \\
C_{s} & D_{s}
\end{array}\right\rfloor\left(\begin{array}{c}
P_{d} \\
Q m_{d}
\end{array}\right)
\end{aligned}
$$

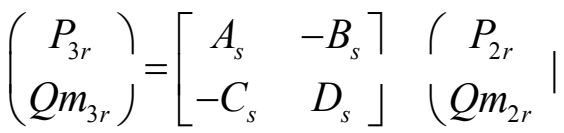

The pressure signals are measured experimentally whereas the mass flow are calculated using the transfer matrices for the tube given by equation (1). The measured transfer matrix characterizes the geometry between the points 2 and 3 and shown in FIGURE 4.
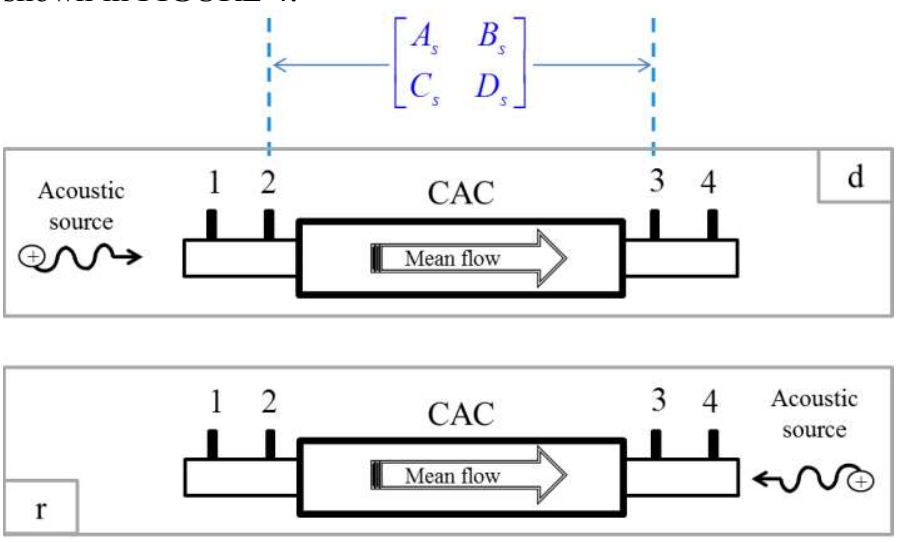

FIGURE 4 - EXPERIMENTAL CHARACTERIZATION OF CAC

Two excitations are possible to measure the transfer matrix of the CAC: either by using the impulse dynamic bench or by measuring the matrix directly on the engine bench.

The advantage of measuring the transfer matrix on the impulse bench is in the nature of the rapid excitation that excites the entire frequency bandwidth in question. However, the excitation on the bench is non-repetitive which makes the signal processing challenging and depending on the geometry upstream and downstream of the CAC, the measured response would be different due to the different resonance profile of the global geometry. Appropriate lengths must be selected upstream and downstream in order to excite the desired frequencies which correspond to the harmonics $m$ of the engine. FIGURE 5 shows for example the CAC on the impulse bench with a $474 \mathrm{~mm}$ long bend between its interface and the impulse bench. The idea is to adjust the length $L_{m}$ between the excitation of the bench and the CAC in a way to excite the desired frequencies. This means that the measured resonant frequencies on the impulse bench of the CAC must verify equation (7) where $N$ is the engine rotational speed in RPM. The length $L_{m}$ is chosen in a way to have the fundamental frequency on the impulse bench $\omega_{1}$ equal to the frequency of the $m^{\text {th }}$ harmonic of the engine. For each length $L_{m}$, a set of resonant frequencies are registered.

$$
\begin{aligned}
& \vec{\omega}_{i}\left(L_{m}\right)=\left[\begin{array}{lllll}
\omega_{1}\left(L_{m}\right) & \ldots & \omega_{i}\left(L_{m}\right) & \ldots & \omega_{n}\left(L_{m}\right)
\end{array}\right] \\
& \omega_{1}\left(L_{m}\right)=\frac{N}{30} \\
& i=1 \rightarrow
\end{aligned}
$$

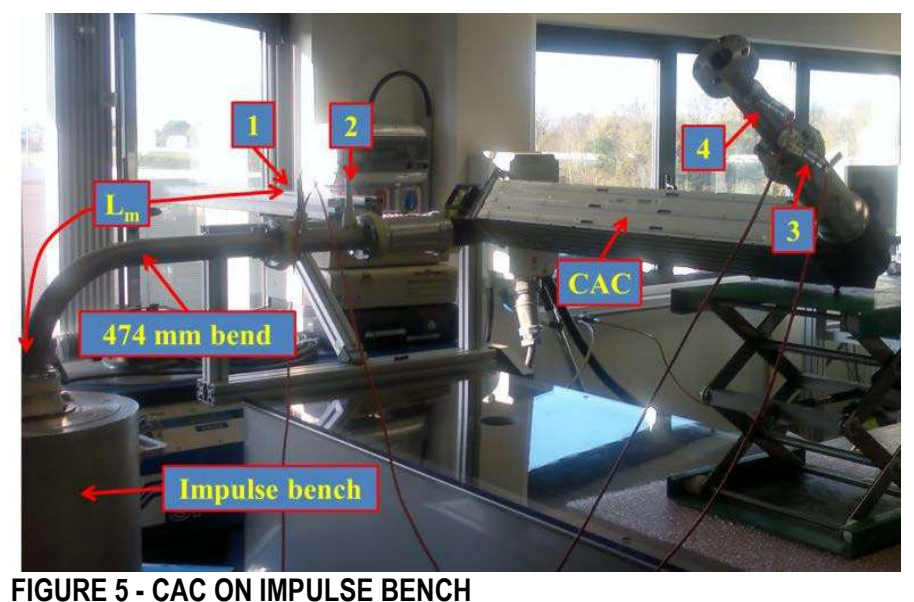

For each length $L_{m}$, a transfer matrix of the CAC is identified. This transfer matrix can be used for an engine rotational speed equal to $N$. The final transfer matrix, which can be used for the entire engine speed range is determined by summing all the cases together using the signal processing method explained by Mezher et al. [36]. The length $L_{m}$ of the bend is used in the direct (d) and reverse (r) configurations.

The transfer matrix can also be measured directly at the intake of the engine as shown in FIGURE 6. The CAC is installed on a turbocharged 1.5L Diesel engine with measuring tubes upstream and downstream.

In this case, the experimental measurements on the engine bench won't be used as usually to validate a $1 \mathrm{D}$ model of the 
CAC but rather to measure the transfer matrix of the CAC which is then used in an engine simulation later on. The advantage of this method is that there is no need to have different lengths on the bench in order to change the resonant frequencies, this is done by simply varying the engine rotational speed and thus the frequency content is enriched this way. Another advantage of the direct measurement on the bench is the fact that the matrix is measured under real conditions of pressure and temperature, and thus non-linearities are minimized when modeling.

In this paper, the transfer matrix is measured on the impulse bench then validated on an engine bench in order to highlight the methodology and directly compare the modeling to experimental data.

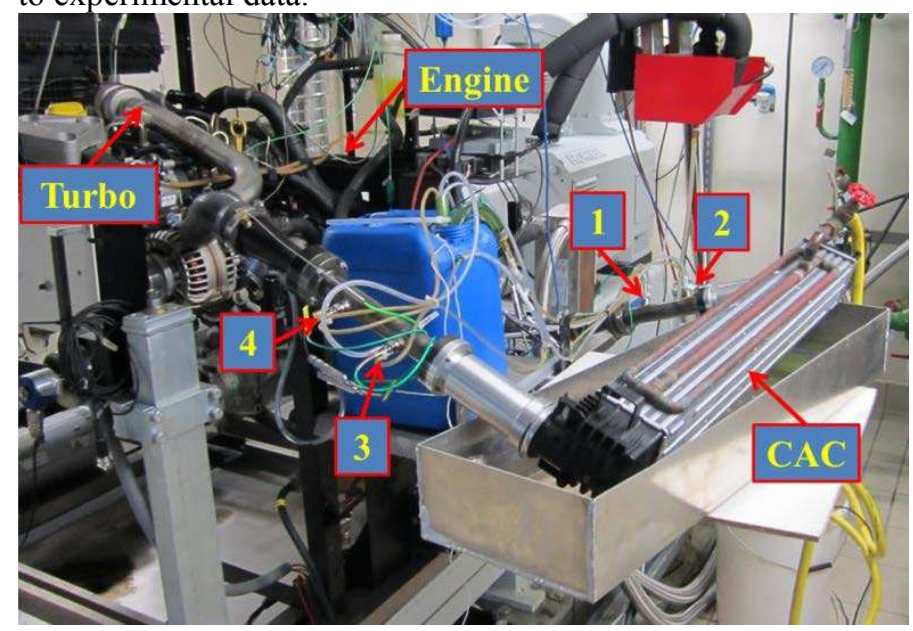

FIGURE 6 - CAC ON ENGINE BENCH

The main objective is to measure on the impulse bench a transfer matrix for the complicated geometry (CAC) and then include this transfer matrix model in a $1 \mathrm{D}$ engine simulation code. The goal is to easily model the CAC geometry and to speed up the time needed to setup a 1D model as well as the simulation time.

\section{VALIDATION}

In this paragraph the measured transfer matrix on the impulse bench will be validated with the experimental data on the engine bench. This is done by comparing:

- The measured pressure at point 3 with the pressure calculated using the transfer matrix approach of the geometry [1-2-3]. This is done by using equation (8).

$$
P_{3 \text { matrix }}=\left|\left(P_{2}-A P_{1}\right) \frac{D}{B}+C P_{1}\right| B_{s}-A_{s} P_{2}
$$

- The dynamic mass flow at point 3 given by equation (9) for the geometry [3-4] with the one given by equation (10)) for the geometry [1-2-3]. Equation (9) is obtained by writing the transfer matrix of the tubular part between point 3 and point 4 in a similar way as equation (1) which is given for the tube [1-2]. Then $\mathrm{Qm}_{3}$ is calculated as a function of the measured pressures $P_{3}$ and $P_{4}$. Equation (10) is obtained by replacing the mass flow $Q m_{2}$ in equation (6) with the mass flow obtained as a function of pressure from equation (1). The results of using equations (9) and (10) is a comparison of the dynamic mass flow at point 3 using two different transfer matrices.

$$
\begin{gathered}
Q m_{3 \text { matrix }[1-2]}=\left(\frac{P_{4}-A P_{3}}{B}\right) \\
\left.Q m_{3 \text { matrix }[1-2-3]}=C_{s} P_{2} \quad D \quad \frac{P_{2}-A P}{B}\right)
\end{gathered}
$$

Once the pressure spectra is calculated using equation (8), an inverse Fast Fourier Transform is performed in order to bring the results back into the time domain. The results are shown in the time domain as function of the engine crank angle in FIGURE 7 for three operating speeds (1500 RPM, 2000 RPM and 3000 RPM). The pressure at point 2 is plotted as well is order to highlight the good transmission and reflective properties of the transfer matrix. FIGURE 8 also gives the results of pressure in the frequency domain by plotting the sound pressure levels (SPL in dB) as defined by Munjal [2].

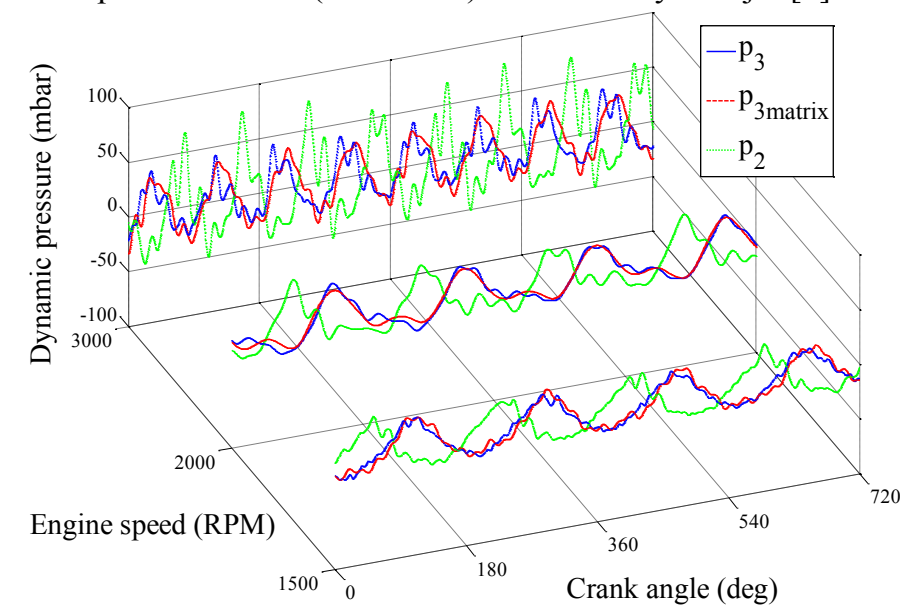

FIGURE 7 - TIME DOMAIN COMPARISON FOR PRESSURE AT POINT 3

The pressure results show a good coherence between the measured pressure and the one calculated using the transfer matrix approach. The engine harmonics at the low frequency region in FIGURE 8 are well reproduced with the transfer matrix. These low frequencies have the most influence on the time domain signals. The dynamic mass flow has also to be checked for consistency, this is because the objective is to have a transfer matrix that can communicate pressure and mass flow variables with the non-linear 1D code. FIGURE 9 gives the dynamic mass flow computed using equations (9) and (10). 


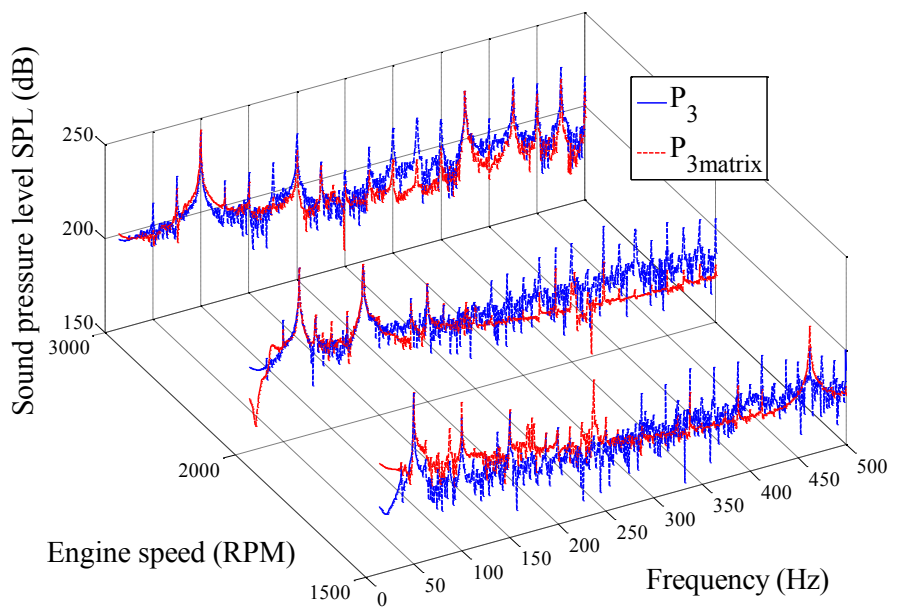

FIGURE 8 - FREQUENCY DOMAIN COMPARISON FOR PRESSURE AT POINT 3

The good coherence between the two methods used to calculate the mass flow indicates that the transfer matrices are capable of taking into account the aero-acoustic coupling between the pressure and the mass flow and are therefore suited to be incorporated in an engine simulation.

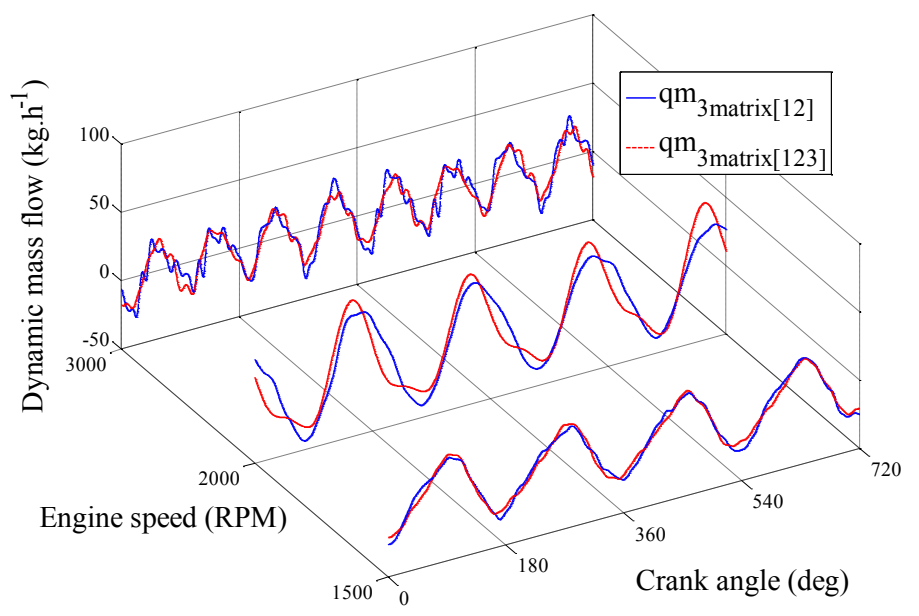

FIGURE 9 - TIME DOMAIN COMPARISON FOR MASS FLOW AT POINT 3

\section{LINEAR FREQUENCY DOMAIN / NON-LINEAR TIME DOMAIN COUPLING}

The pressure measurements on the dynamic bench were done using a sampling frequency $F S=20 \mathrm{kHz}$. The transfer matrix poles are identified as frequency domain vectors that have the same length as the FFT used.

The objective is engine simulation by integrating the linear transfer matrix into an engine simulation code. GT-Power ${ }^{\circledR}$ is chosen; it is a 1D non-linear time domain resolution of the unsteady gas dynamics equations. The problem then becomes how to couple the frequency domain information with a time domain code. Work has been done to couple an impedance model to a time domain code by using the method of characteristics [25] [26] or by using the transfer function approach described in this paper to link GT-Power ${ }^{\circledR}$ to
Simulink ${ }^{\circledR}$ [4] [29] [30]. These represent impedance models where the pressure and mass flow are characterized in a single point for a given intake line. Portier et al. [20] implemented the measured transfer matrix into an acoustic simulation code, however the computation remains solely in the frequency domain. Alberston et al. [37] tested existing approaches to couple a non-linear time domain source description to a linear frequency domain model on a simple valveless one cylinder cold engine. It was found that as the amplitude levels and the degree of nonlinearity are increased, the solutions slowly diverge. Reasons for these discrepancies could be due to the way the sampled discrete values are implemented. It was also found that when an Inverse Fast Fourier Transform is used to output values over a short period of time, high frequencies can arise. They are problematic in time / frequency coupling because when high frequencies are present in an inverse Fourier transform, Gibb's phenomenon will arise. These spurious oscillations do not exist in the physical system, but they arise from the mathematics. Knutsson et al. [27] introduced a technique where the transfer matrix was coded as FIR elements in a Simulink ${ }^{\circledR}$ code, the latter communicated pressure to GTpower ${ }^{\circledR}$ on both sides of the matrix. GT-Power ${ }^{\circledR}$ was responsible for calculating the mass flow and the coupled simulation was done in this way. This enabled the authors to couple a frequency model to a time domain resolution scheme. The results were promising for frequencies above $100 \mathrm{~Hz}$, but were less precise for the low frequency region. Also the measurements and simulation were done in acoustic conditions, i.e. zero mean flow.

The proposed methodology consists on coupling the transfer matrix to GT-Power ${ }^{\circledR}$ using a similar approach. The dynamic and static components will be treated apart as highlighted by equation (1). The transfer matrix is responsible for calculating the dynamic component whereas a pressure loss coefficient for the charge air cooler calculates the mean pressure. The matrix in equation (6) can be re-written in the following form:

$$
\left(\begin{array}{c}
P_{2} \\
Q m_{3}
\end{array}\right)_{j \omega}=\left(\begin{array}{llll}
M T_{1} & M T_{2} & \left(P_{3}\right. \\
M T_{3} & M T_{4} & j \omega & \left(Q m_{2}\right.
\end{array}\right)_{j \omega}
$$

The objective of writing the matrix in the form given by equation (11) is to exchange both pressure and mass flow information with GT-Power $($ : mass flow at point 2 and pressure at point 3 are taken from the time domain code and are used to calculate in the frequency domain the pressure at point 2 and the mass flow at point 3 . The calculated variables are then injected back into the time domain code. The discrete functions $M T(j \omega)$ are transformed into continuous functions using a least square frequency identification procedure. A polynomial fitting is made by calculating the parameters of equation (12) where $q$ is the discrete variable for the Z-

Transform and $n_{a} \geq n-1$. 


$$
\operatorname{MT}\left(q=\frac{b_{0}+b_{1} q^{1}+b_{2} q^{-2}+\ldots+b_{n_{b}-1} q^{-\left(n_{b}-1\right)}}{a_{0}+a_{1} q^{-1}+a_{2} q^{-2}+\ldots+a_{n_{a}} q^{-n_{a}}}\right.
$$

In this way the measured transfer matrix poles are transformed into the continuous domain and implemented in Simulink ${ }^{\circledR}$ as IDPLOY objects or transfer functions. FIGURE 10 resumes the coupling scheme where the CAC element from FIGURE 1 is replaced with a Simulink ${ }^{\circledR}$ component in GTPower ${ }^{\circledR}$.

As shown in FIGURE 10, the absolute mass flow is taken from the GT-Power model at point 2 (intake plenum side) as well as the absolute pressure at point 3 (CAC inlet). The mean mass flow value from point 2 is transmitted across the CAC into point 3 (continuity equation) and its value is used to calculate the pressure drop attributed to the mean pressure at point 3 . The static components of pressure at point 2 and mass flow at point 3 are thus calculated.

The transfer matrix poles then calculate the dynamic pressure at point 2 and mass flow at point 3 (equation (11)). The GT-Power ${ }^{\circledR}$ model is split into two halves (upstream and downstream of the CAC) and is responsible for calculating pressure and mass flow upstream of the intake valves and thus evaluates engine filling and volumetric efficiency.

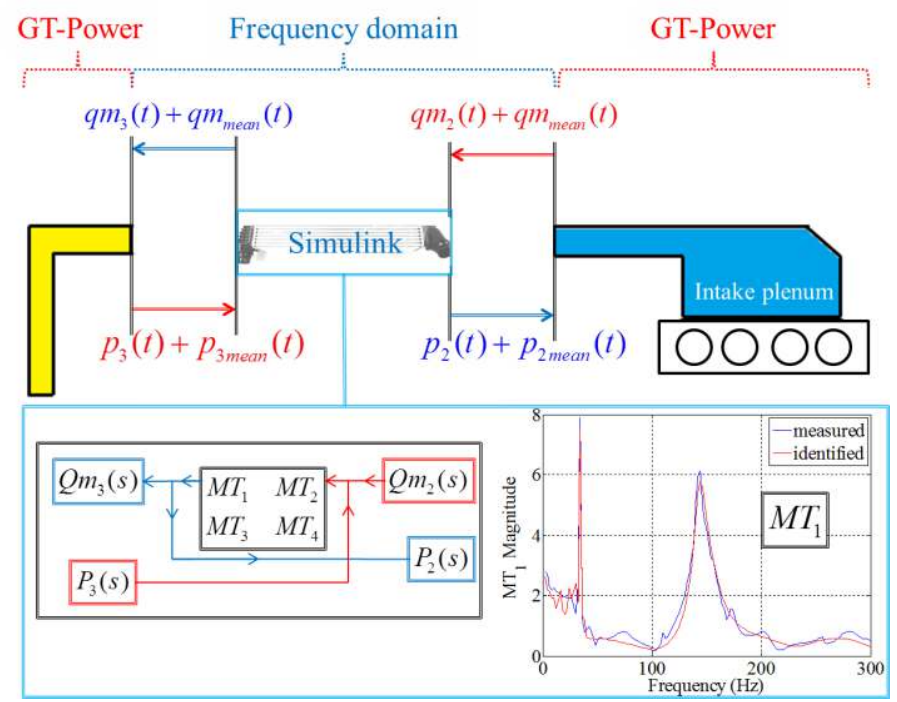

FIGURE 10 - COUPLING SCHEME SIMULINK / GT-POWER

\section{RESULTS}

In order to validate the coupling, the $1.5 \mathrm{~L}$ Diesel engine used in FIGURE 6 is coupled to an electric motor and run without combustion. The engine is thus used as a pulse generator. The CAC is installed at the intake of the engine with rigid constant cross section tubes; this is done in order to keep the intake line as simple as possible for modeling in GTPower ${ }^{\circledR}$. Because the engine is run without combustion, the boost pressure normally provided by the enthalpy of the exhaust gases is assured by using an independent hot gas stand. The latter is used to insure the boost pressure mapping at the intake. FIGURE 11 shows the schematic of the setup: a $1.5 \mathrm{~m}$ tube is used between the plenum and the CAC outlet whereas a $0.9 \mathrm{~m}$ tube is used between the compressor outlet and CAC. The internal diameter is $28 \mathrm{~mm}$. Rigid tubes are used because they are easier to model in GT-Power ${ }^{\circledR}$; this way any discrepancies between the coupled simulation and the measurements will be due to the transfer matrix methodology and not to errors of modeling complex pipes and elements in GT-Power ${ }^{\circledR}$.

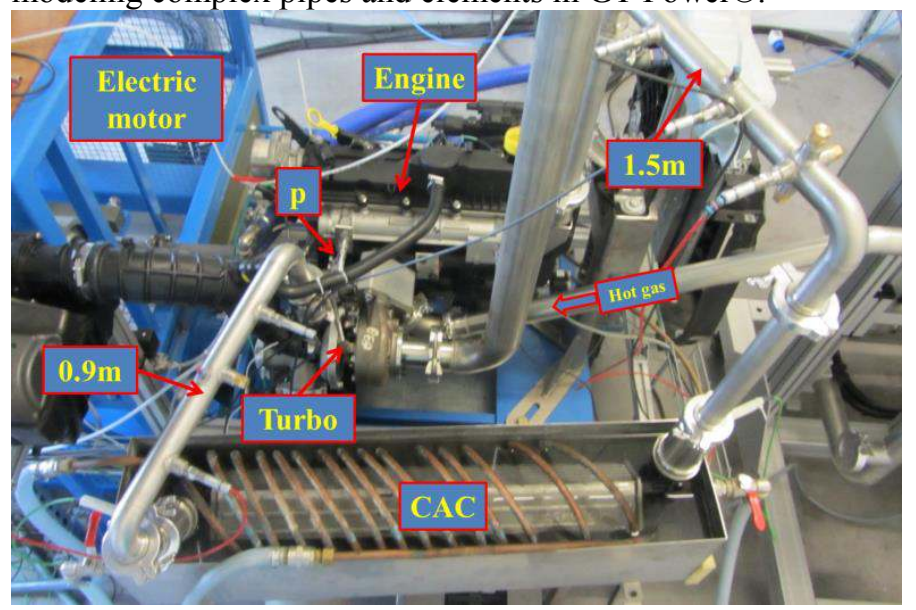

FIGURE 11 - ELECTRIC DRIVEN ENGINE WITH CAC

The CAC was cooled using sprayed water in order to have an exit air temperature of around $50^{\circ} \mathrm{C}$. Instantaneous pressure is measured in a designated point in the intake plenum. This pressure will be used to compare measurements and simulation results. FIGURE 12 represents the GT-Power ${ }^{\circledR}$ model where the engine block, cylinder head, intake plenum and exhaust manifold are modeled using 1D tubular elements. A subassembly element in GT-Power contains the Simulink ${ }^{\circledR}$ block (SimulinkHarness element) with the associated pressure sensors and boundary conditions elements. The temperature gradient across the $\mathrm{CAC}$ was forced using wall heat exchange and the outlet temperature was fixed to $50^{\circ} \mathrm{C}$. Care must be taken when setting up the simulation parameters; Simulink ${ }^{\circledR}$ must be in exact synchronization with the GT-Power ${ }^{\circledR}$ model, maximum time steps have to be chosen with respect to the sampling frequency used to measure the transfer matrix poles. A variable time step ODE45 (Dormand-Prince) solver is chosen in Simulink ${ }^{\circledR}$ and is forced into the GT-Power ${ }^{\circledR}$ model. The meshing in GT-Power ${ }^{\circledR}$ varies between $10 \mathrm{~mm}$ and $30 \mathrm{~mm}$ depending of the pipe in question.

FIGURE 12 represents a simplified model of the engine, where the compressor and turbine were replaced with simple pressure and temperature boundary conditions. This is because the main objective here is to use the experimental/numerical setup as a pulse generator. The simulation was run for 4 seconds. 


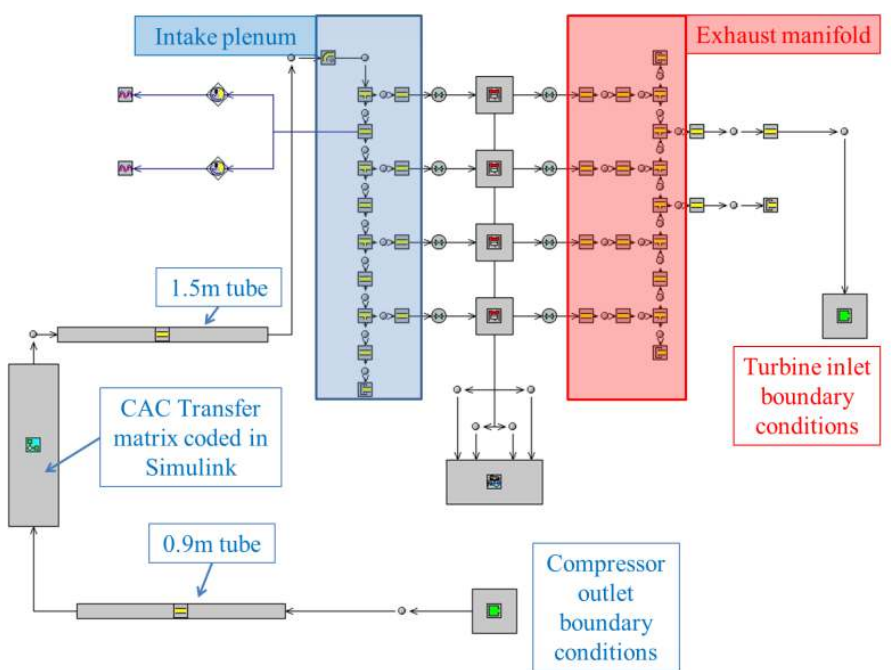

FIGURE 12 - SIMPLIFIED GT-POWER ENGINE MODEL WITH TRANSFER MATRIX OF CHARGE AIR COOLER CODED IN SIMULINK

FIGURE 13 compares the measured and simulated pressure traces at the intake for an operating speed of 1500 RPM.

The results show a good correlation mainly for the frequency response. The simulated pressure signal seems to be underestimated when compared in detail to the experimental pressure data. FIGURE 14 shows the pressure response for an engine operating speed of 2000 RPM.

Good coherence is generally obtained, with some more prominent discrepancies between the measured and simulated signals. This can be explained by the three following reasons:

- The linear assumptions of a transfer matrix.

- The fact that the heat transfer effect across the CAC was not taken into account when the transfer matrix was identified. The heat gradient across the CAC can have a significant effect of the propagation properties of the air and therefore would influence the wave action at the intake through modifying the reflection at the CAC's interface.

- The identification procedure itself given by the equation (12) where a least square method is used. Information is undoubtedly lost when attempting this kind of identification because of the difficulty to correctly fit the data throughout the entire frequency range without overcomplicating the polynomial functions.

Nevertheless, good correlation is observed which indicates that the transfer matrix methodology proposed here can be used for engine simulation purposes because it takes into account the aero-acoustic coupling effects that are most prominent at low frequencies.
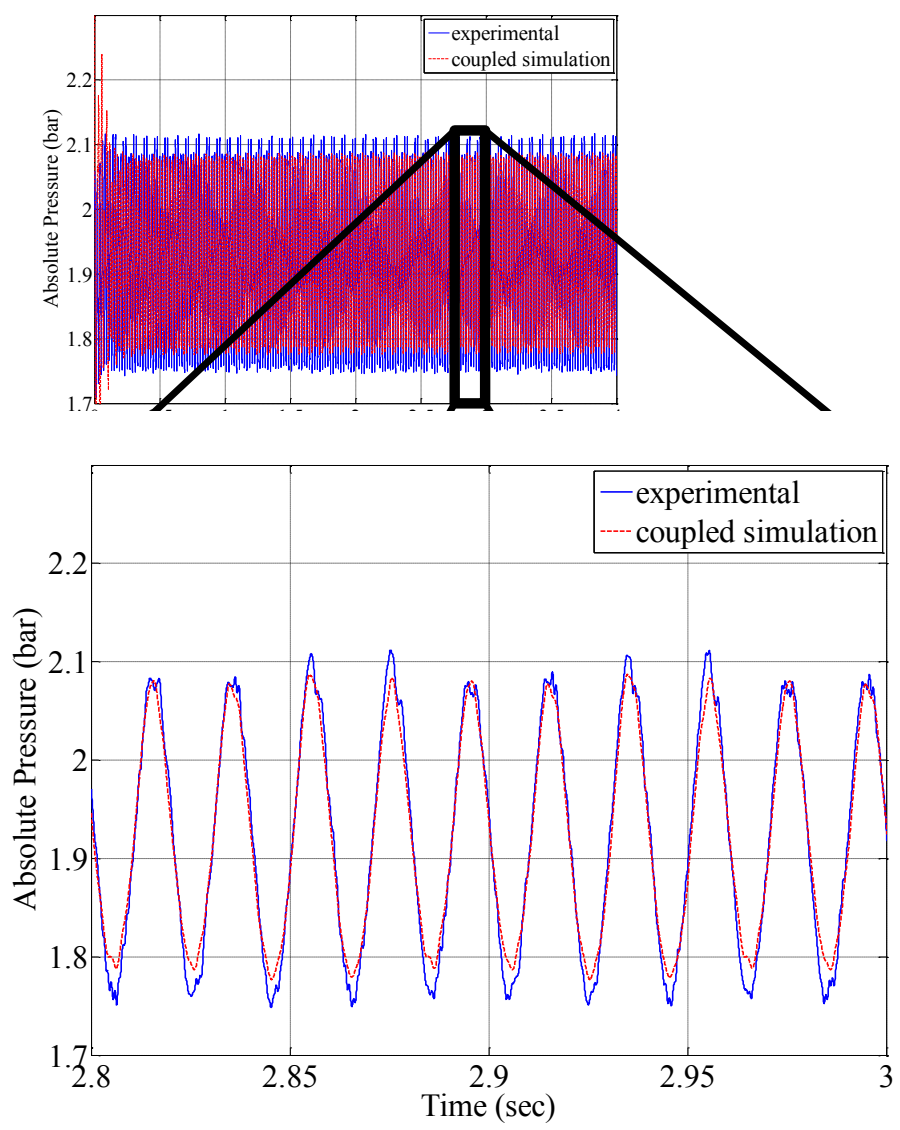

rIGUKE 13 - MEASUKEU ANU SIMULAIEU PKESSUKE AI 13UU RPIM

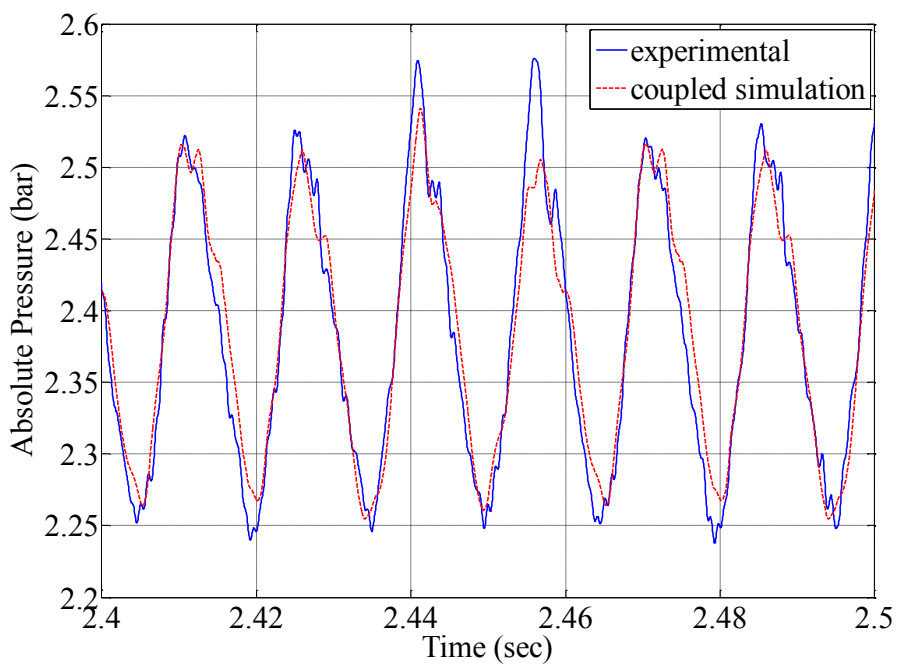

FIGURE 14 - MEASURED AND SIMULATED PRESSURE AT 2000 RPM

\section{CONCLUSIONS}

The transfer matrix methodology proposed in this article was validated for a simple tube then applied to a more complicated geometry, a charge air cooler. It consists of characterizing pressure and mass flow variables on an impulse flow bench using a mass flow excitation similar to that encountered at the intake of the engine. It remains a linearized model however it takes into account the coupled effects 
between the mass flow and the pressure. The transfer matrix for the $\mathrm{CAC}$ was validated by measuring the pressure traces at the intake of a 4 cylinder turbocharged 1.5L Diesel engine. Good correlation for pressure and mass flow was observed. Thus the transfer matrix can be incorporated into an engine simulation code. This was done by identifying parameters for polynomial expressions for each transfer matrix pole. They were coded in Simulink ${ }^{\circledR}$ which communicated pressure and mass flow information with GT-Power ${ }^{\circledR}$. Simulated pressure traces were compared to measured ones at the intake of an electrically driven engine (pulse generator). Results show good correlation. However there is room for improvement mainly by modeling or measuring the effects of a heat gradient across the CAC and by improving the coupling procedure. The overall objective is a methodology that replaces a complicated element (such as a CAC) with a transfer matrix model. The latter is measured and would be precise enough for the low frequency region relative to engine filling where the aero-acoustic effects are important. This would reduce the computational times but also the time needed to model and setup experimental validation for a $1 \mathrm{D}$ modeling solution.

\section{NOMENCLATURE}

\begin{tabular}{|c|c|}
\hline$a, b$ & Identified parameters \\
\hline$A, B, C, D$ & Transfer matrix poles for the simple tube \\
\hline$A_{s}, B_{s}, C_{s}, D_{s}$ & Transfer matrix poles for the CAC \\
\hline$c_{0}$ & Speed of sound $\left(\mathrm{m} \cdot \mathrm{s}^{-1}\right)$ \\
\hline$d$ & Direct configuration \\
\hline FFT & Fast Fourier transform \\
\hline$F S$ & Sampling frequency $(\mathrm{kHz})$ \\
\hline$i$ & Resonant mode number \\
\hline i & Imaginary constant \\
\hline$L$ & Length (m) \\
\hline$m$ & Engine harmonic \\
\hline$M T$ & Transfer matrix poles used for coupling \\
\hline$n$ & Number of identified harmonics \\
\hline$n, n_{b}$ & Denominator and numerator orders \\
\hline$N$ & Engine rotational speed (RPM) \\
\hline$p$ & Dynamic fluctuating pressure (mbar) \\
\hline$p_{\text {absolute }}$ & Absolute pressure (mbar) \\
\hline$p_{\text {mean }}$ & Mean pressure value (mbar) \\
\hline$P$ & Dynamic pressure spectra \\
\hline$P F_{x}$ & Transfer function for pressure at point $x$ \\
\hline$q$ & Discrete Z-transform variable \\
\hline$q m$ & Dynamic fluctuating mass flow $\left(\mathrm{kg} \cdot \mathrm{h}^{-1}\right)$ \\
\hline$q m_{e x c}$ & Mass flow excitation on the bench $\left(\mathrm{kg} \cdot \mathrm{h}^{-1}\right)$ \\
\hline
\end{tabular}

\begin{tabular}{|c|c|}
\hline$Q F_{x}$ & Transfer functions for mass flow \\
\hline$Q m$ & Dynamic mass flow spectra \\
\hline$Q m_{e x c}$ & Mass flow excitation spectra \\
\hline$r$ & Inverse configuration \\
\hline$S$ & Cross section of pipe $\left(\mathrm{m}^{2}\right)$ \\
\hline$T F$ & Transfer function of the intake $\left(\mathrm{mbar} \cdot \mathrm{kg}^{-1} \cdot \mathrm{h}\right)$ \\
\hline$x$ & Abscissa of the tube $(\mathrm{m})$ \\
\hline$Z_{c}$ & Characteristic impedance of the pipe $\left(\mathrm{m}^{-1} \cdot \mathrm{s}^{-1}\right)$ \\
\hline$\omega$ & Angular frequency $\left(\right.$ rad. $\left.\mathrm{s}^{-1}\right)$ \\
\hline$\epsilon$ & Damping parameter \\
\hline
\end{tabular}

\section{ACKNOWLEDGMENTS}

The author would like to thank the Internal Combustion Engine team at École Centrale de Nantes and the Thermodynamics and Acoustic departments in the advanced development at Mann + Hummel France.

\section{REFERENCES}

[1] WINTERBONE, D.E.; PEARSON, R.J., Theory of Engine Manifold Design: Wave Action Methods for IC Engines.: Professional Engineering Publishing, 2000.

[2] MUNJAL, M.L., Acoustics of ducts and mufflers with application to exhaust and ventilation system design.: John Wiley \& Sons, 1987.

[3] MEZHER, H.; CHALET, D.; MIGAUD, J.; CHESSE, P.; RAIMBAULT, V., Transfer matrix computation wave action simulation in an internal combustion engine ASME 2012 11th Biennal Conference on Engineering Systems Design and Analysis. Nantes, France: ESDA201282579, 2-4 July 2012.

[4] CHALET, D.; MAHE, A.; MIGAUD, J.; HETET, J.-F., Multi-frequency modelling of unsteady flow in the inlet manifold of an internal combustion engine.: Proceedings of the Institution of Mechanical Engineers, Part D: Journal of Automobile Engineering, Vol. 226(5), pp. 648-658, DOI: 10.1177/0954407011423745, May 2012.

[5] CHALET, D.; CHESSE, P., Fluid dynamic modeling of junctions in internal combustion engine inlet and exhaust systems.: Journal of Thermal Science, Vol. 19(5), pp. 410418, DOI: 10.1007/s11630-010-0402-0, 2010.

[6] BENSON, R.S., The Use of Generalized Boundary Conditions for Devices in Non-steady Flow in One Dimensional Pipe Systems.: Int. J. Mech. Sci, Vol. 21, pp. 399-406, 1979.

[7] SEIFERT, H., 20 Jahre Erfolgreiche Entwicklung des Programmsystems PROMO.: MTZ Motortech. Z., Vol. 11, pp. 478-488, 1990.

[8] CORNOLTI, L.; ONORATI, A.; CERRI, T.; 
MONTENEGRO, G.; PISCAGLIA, F., $1 D$ simulation of a turbocharged Diesel engine with comparison of short and long EGR route solutions.: Applied Energy, Vol. 111, pp. 1-15, DOI: 10.1016/j.apenergy.2013.04.016, 2013.

[9] MONTENEGRO, G.; ONORATI, A.; DELLA TORRE, A., The prediction of silencer acoustical performances by $1 D, \quad 1 D-3 D$ and quasi-3D non-linear approaches.: Computers \& Fluids, vol. 71, pp. 208-223, 2013.

[10] MONTENEGRO, G.; DELLA TORRE, A.; ONORATI, A.; FAIRBROTHER, R., A Nonlinea rQuasi-3DApproach for the Modeling of Mufflers with Perforated Elements and Sound-Absorbing Material.: Advances in Acoustics and Vibration, Hindawi Publishing Corporation, Vol. 2013, Article ID 546120, 2013.

[11] WALLACE, F.J.; BOXER, G., Wave action in diffusers for exhaust-pipe systems, with special reference to the scavenging of two-stroke engines.: Proceedings of the Institution of Mechanical Engineers, Vol. 170(1), pp. 11311156, DOI: 10.1243/PIME_PROC_1956_170_094_02, June 1956.

[12] CHEN, C.; VESHAGH, A.; WALLACE, F., A comparison between alternative methods for gas flow and performance prediction of internal combustion engines. Milwaukee: SAE technical paper 921734, DOI: 10.4271/921734, 1992.

[13] PAYRI, F.; TORREGROSA, A.J.; PAYRI, R., Evaluation through pressure and mass velocity distributions of the linear acoustical description of I. C. engine exhaust systems.: Applied Acoustics Elsevier, Vol. 60(4), pp. 489504, DOI: 10.1016/S0003-682X(00)00006-2, 2000.

[14] PEARSON, R.J.; WINTERBONE, D.E., A rapid wave action simulation technique for intake manifold design. Detroit, MI: SAE technical paper 900676, DOI: 10.4271/900676, 1990.

[15] ELNEMR, Y., Acoustic Modeling and Testing of Exhaust and Intake System Components. Stockholm, Sweden: Thèse, The Marcus Wallenberg Laboratory of Sound and Vibration Research, Department of Aeronautical and Vehicle Engineering, 2011.

[16] GIBIAT, V.; LALOË, F., Acoustical impedance measurements by the two-microphone-three-calibration (TMTC) method.: Journal Acoustical Society of America, Vol88(6), pp. 2533-2545, DOI:10.1121/1.399975, 1990.

[17] BRUNEAU, M., Manuel d'acoustique fondamentale. Paris: Hermes, 1998.

[18] KNUTSSON, M.; ABOM, M., Sound propagation in narrow tubes including effects of viscothermal and turbulent damping with application to charge air coolers.: Journal of Sound and Vibration, Vol. 320, pp. 289-321, DOI:10.1016/j.jsv.2008.07.006, 2009.

[19] TIIKOJA, H., Acoustic Characterization of Turbochargers and Pipe Terminations. Stockholm, Sweden: Thèse, The Marcus Wallenberg Laboratory for Sound and Vibration
Research - Department of Aeronautical and Vehicle Engineering, 2012.

[20] PORTIER, E.; LEANDRE, J., Experimental determination and prediction of turbocharger transfer matrix. Le Mans: SIA technical articles no. R-2012-06-15, 2012.

[21] OHATA, A.; ISHIDA, Y., Dynamic Inlet Pressure and Volumetric Efficiency of Four Cycle Cylinder Engine.: SAE etchnical paper no.820407, 1983.

[22] MATSUMOTO, I.; OHATA, A., Variable induction systems to improve volumetric efficiency at low and/or medium engine speeds.: SAE no.860100, 1986.

[23] WINTERBONE, D.E.; PEARSON, R.J., Design Techniques for Engine Manifolds.: Professional Engineering Publishing, 1999.

[24] HARRISON, M.F.; STANEV, P.T., A linear acoustics model for intake wave dynamics in IC engines.: Journal of Sound and Vibration, Vol. 269(1-2), pp. 361-387, DOI: 10.1016/S0022-460X(03)00196-2, 6 January 2004.

[25] HARRISON, M.F.; DE SOTO, I.; RUBIO UNZUETA, P., A linear acoustic model for multi-cylinder IC engine intake manifolds including the effects of the intake throttle, Journal of Sound and Vibration 278, 975-1011.: Journal of Sound and Vibration, Vol. 278(4-5), pp.975-1011, DOI:10.1016/j.jsv.2003.12.009, 22 December 2004.

[26] SATHYANARAYANA, Y.; MUNJAL, M.L., A hybrid approach for aeroacoustic analysis of the engine exhaust system.: Applied Acoustics, Vol. 60(4), pp. 425-450, 2000.

[27] KNUTSSON, M.; LENNBLAD, J.; BODEN, H.; ABOM, M., A study on acoustical time-domain two-ports based on digital filters with application to automotive air intake systems.: SAE technical poper no. 2011-01-1522, DOI:10.4271/2011-01-1522, 2011.

[28] P. FONTANA and B. HUURDEMAN, A new evaluation method for the thermodynamic behavior of air intake systems.: SAE 2005-01-1136, 2005.

[29] CHALET, D.; MAHE, A.; MIGAUD, J.; HETET, J.F., $A$ frequency modeling of the pressure waves in the inlet manifold of internal combustion engine.: Applied Energy, Vol. 88(9), pp. 2988-2994, ISSN 0306-2619, DOI 10.1016/j.apenergy.2011.03.036, 2011.

[30] CHALET, D.; MAHE, A.; HETET, J-F.; MIGAUD, J., $A$ new modeling approach of pressure waves at the inlet of internal combustion engines.: Journal of Thermal Science, Vol. 20(2), pp. 181-188, DOI: 10.1007/s11630-011-0455$8,2011$.

[31] MEZHER, H.; MIGAUD, J.; CHALET, D.; RAIMBAULT, V.; CHESSE, P.; HUURDEMAN, B., Transfer matrix computation for intake elements with large pressure fluctuations under mean flow conditions. Detroit, Michigan: SAE 2012 World Congress, SAE2012-01-0672, 24-26 april 2012.

[32] MUNJAL, M.L.; DOIGE, A.G., Symmetry of One- 
Dimensional Dynamical Systems in Terms Of Transfer Matrix Parameters.: Journal of Sound and Vibration, Vol. 163(3), pp. 467-475, DOI: 10.1016/0022-460X(90)90457B, 1990.

[33] RAMMAL, H.; ABOM, M., Acoustics of turbochargers.: SAE no. 2007-01-2205, DOI: 10.4271/2007-01-2205 , 2007.

[34] TO, C.W.S; DOIGE, A.G., The application of transient testing methods to the determination of acoustic properties of unknown system.: Journal of Sound and Vibration, Vol. 71 (4), pp. 545-554, DOI: 10.1016/0022-460X(80)90726$9,1980$.

[35] MUNJAL, M.L.; DOIGE, A.G., Theory of a Two SourceLocation Method for Direct Experimental Evaluation of the Four-Pole Parameters of an Aeroacoustic Element:: Journal Of Sound And Vibration, Vol. 141(2), pp. 323-333, 1990.

[36] MEZHER, H.; CHALET, D.; MIGAUD, J.; RAIMBAULT, V.; CHESSE, P., Transfer matrix measurements for studying intake wave dynamics applied to charge air coolers with experimental engine validation in the frequency domain and the time domain.: Proceedings of the Institution of Mechanical Engineers, Part D: Journal of Automobile Engineering, DOI: 10.1177/0954407012474630, May 21, 2013.

[37] ALBERSTON, F.; BODEN, H.; GILBERT, J., Comparison of different methods to couple nonlinear source descriptions in the time domain to linear system descriptions in the frequency domain - Application to a simple valveless one-cylinder cold engine.: Journal of Sound and Vibration, Vol. 291, pp. 963-985, doi:10.1016/j.jsv.2005.07.046, 2006. 